\title{
Very low amounts of glucose cause repression of the stress-responsive gene HSP12 in Saccharomyces cerevisiae
}

\author{
Ellen de Groot, Jan-paul Bebelman, Willem H. Mager and Rudi J. Planta
}

Author for correspondence: Willem H. Mager. Tel: +31 20444 7569. Fax: +31204447553.

e-mail:mager@chem.vu.nl

Department of Biochemistry and Molecular Biology, IMBW, BioCentrum Amsterdam, Vrije Universiteit, De Boelelaan 1083, 1081 HV Amsterdam, The Netherlands
Changing the growth mode of Saccharomyces cerevisiae by adding fermentable amounts of glucose to cells growing on a non-fermentable carbon source leads to rapid repression of general stress-responsive genes like HSP12. Remarkably, glucose repression of HSP12 appeared to occur even at very low glucose concentrations, down to $\mathbf{0 . 0 0 5} \%$. Although these low levels of glucose do not induce fermentative growth, they do act as a growth signal, since upon addition of glucose to a concentration of $0.02 \%$, growth rate increased and ribosomal protein gene transcription was up-regulated. In an attempt to elucidate how this type of glucose signalling may operate, several signalling mutants were examined. Consistent with the low amounts of glucose that elicit HSP12 repression, neither the main glucose-repression pathway nor CAMP-dependent activation of protein kinase A appeared to play a role in this regulation. Using mutants involved in glucose metabolism, evidence was obtained suggesting that glucose 6-phosphate serves as a signalling molecule. To identify the target for glucose repression on the promoter of the HSP12 gene, a promoter deletion series was used. The major transcription factors governing (stress-induced) transcriptional activation of HSP12 are Msn2p and Msn4p, binding to the general stress-responsive promoter elements (STREs). Surprisingly, glucose repression of HSP12 appeared to be independent of Msn2/4p: HSP12 transcription in glycerol-grown cells was unaffected in a Amsn2 Amsn4 strain. Nevertheless, evidence was obtained that STRE-mediated transcription is the target of repression by low amounts of glucose. These data suggest that an as yet unidentified factor is involved in STRE-mediated transcriptional regulation of HSP12.

Keywords: glucose repression, signal transduction, protein kinase A (PKA), glucose 6phosphate, HSP12

\section{INTRODUCTION}

Cells of Saccharomyces cerevisiae possess the ability to respond rapidly and effectively to changing conditions of the surrounding medium. Indeed, adequate perception of environmental signals and subsequent efficient signal transduction is essential for survival and for proper adjustment to new growth demands. As part of these adaptations to changing growth conditions, the expression level of many yeast genes is modified. When yeast culture conditions become more favourable, tran-

Abbreviations: FGM, fermentable growth medium (pathway); PKA, protein kinase A; STRE, stress-responsive element. scription of growth-related genes like the ribosomal protein genes is induced, whereas under stress exposure the expression level of the same genes is decreased (Mager \& Planta, 1991). Conversely, stress-responsive genes are highly expressed under adverse growth conditions and down-regulated during normal growth (Hohmann, 1997).

We have studied expression levels of the general stressresponsive gene HSP12 under various growth conditions. This gene, encoding a small heat-shock protein, is expressed under conditions of high osmolarity, heat shock and oxidative stress, during nutrient limitation (Praekelt \& Meacock, 1990; Varela et al., 1995) and during growth on non-fermentable carbon sources 
(Siderius et al., 1997). The transcriptional regulators Msn2p and Msn4p are involved in the activation of HSP12 expression during stress conditions (MartinezPastor et al., 1996). These factors have been shown to shuttle between cytosol and nucleus in conditions allowing normal growth, and to accumulate in the nucleus in stress conditions and under circumstances of low protein kinase A (PKA) activity (Gorner et al., 1998). Msn2p and Msn4p bind to stress-responsive elements (STREs) that are present in the promoter of many stress-responsive genes (Moskvina et al., 1998) and mediate transcriptional activation of these genes. The promoter of the HSP12 gene contains five of these elements, which have been shown to be involved in transcriptional regulation under different stress conditions (Varela et al., 1995; Siderius et al., 1997).

We have studied repression of HSP12 expression under conditions in which the growth potential of the cell increases, by performing carbon-source-shift experiments. Cells growing on a non-fermentable carbon source contain a high level of HSP12 mRNA (Siderius $e t$ al., 1997), which is rapidly diminished when glucose is added to the culture.

Glucose-dependent repression of many genes is regulated via the main glucose-repression pathway. This signalling route is involved in repression of genes involved in the use of alternative carbon sources, and of gluconeogenic genes (Ronne, 1995; Gancedo, 1998). A binding site for the transcription factor Mig1p is present in the promoter of these genes (Nehlin et al., 1991; Lundin et al., 1994). This factor binds to a complex of co-repressors containing Ssn6p and Tup1p, and the total complex confers glucose repression (Treitel \& Carlson, 1995).

During the transition from vegetative growth to fermentation, the activation of PKA results in adaptive changes in enzyme activities and gene expression (Thevelein, 1994). PKA is a multisubunit protein kinase consisting of a pair of regulatory subunits encoded by $B C Y 1$ and a pair of catalytic subunits redundantly encoded by TPK1, TPK2 and TPK3 (Thevelein, 1994). Addition of glucose to a non-fermenting yeast culture results in activation of adenylate cyclase, causing a rapid increase in the cellular level of cAMP. This compound binds to the regulatory subunit of PKA, thereby releasing and activating the catalytic subunits. It is still unclear by what mechanism addition of glucose causes activation of adenylate cyclase. It has been proposed that Ras $2 p$ is involved in this process (Jiang et al., 1998). New evidence has shed some doubt on this (Colombo et al., 1998), since it was shown that not Ras2p but rather the G-protein $\alpha$-subunit Gpa2p and the G-protein-coupled receptor Gpr1p are involved in the increase in cellular cAMP after glucose addition (Yun et al., 1998; Kraakman et al., 1999). Ras2p may be indirectly involved in glucose activation of PKA since it mediates activation of adenylate cyclase upon the intracellular acidification that occurs after addition of sugar to yeast cells (Colombo et al., 1998).
An alternative way of activating PKA, independent of the cellular cAMP level, has been described; this pathway is called the fermentable growth medium (FGM) induced pathway (Thevelein, 1991; Crauwels et al., 1997). Phosphorylation of glucose is not required for activation of PKA via this route, in contrast to activation via cAMP (Pernambuco et al., 1996). The TPK homologue protein kinase $S \mathrm{ch} 9 \mathrm{p}$ is believed to play a role in this pathway (Crauwels et al., 1997).

In the present study, we used mutants defective in several glucose signalling components to investigate which signal transduction routes play a part in the negative regulation of HSP12 expression by low amounts of glucose. In addition, we used reporter constructs containing different regions of the HSP12 promoter to map promoter elements involved in glucose repression.

\section{METHODS}

Yeast growth. Yeast cultures were grown on YNB/glycerol medium $(0.67 \%$ yeast nitrogen base without amino acids, $5 \%$ glycerol, necessary amino acids, $0.02 \%$ glucose) or $\mathrm{YP} /$ ethanol medium ( $1 \%$ yeast extract, $2 \%$ peptone, $1 \%$ ethanol, necessary amino acids, $0.02 \%$ glucose). Genotypes of the strains used in this work are listed in Table 1. HSP12 promoter-GUS fusions containing 606 (KV3-GUS), 234 $(\Delta 32-$ GUS $)$ and 150 ( $\Delta 31-$ GUS) bp of the $5^{\prime}$ flanking region of the HSP12 gene, respectively, were inserted in the URA3 locus of W303-1A cells. Single-copy integration was checked by Southern blot analysis.

Carbon-source-shift experiments. Cells were grown to an $\mathrm{OD}_{660}$ of $0 \cdot 2-0 \cdot 3$ (analysed on Beckman DU-6Z spectrophotometer). At zero time, glucose, fructose or 6-deoxyglucose was added in various concentrations. Samples were taken after 10, 30, 60 and $90 \mathrm{~min}$ and immediately frozen in liquid nitrogen. Total RNA was isolated as described by Toda et al. (1985) and analysed by Northern blot hybridization as described by Varela et al. (1995). DNA probes were labelled using the random priming method (Prime-a-Gene labelling system; Promega). Actin signals were used as a loading control and signal quantification was performed by phosphoimaging (Phosphoimager 425; Molecular Dynamics). Relative HSP12 mRNA levels were calculated by dividing the quantified HSP12 signal by the ACT1 signal. The relative HSP12 signal at time zero was defined as 1 , and the HSP12 levels at other time points are given relative to this starting level.

\section{RESULTS}

\section{Carbon-source-dependent regulation of HSP12 gene expression}

Transfer of $S$. cerevisiae from a growth medium with a non-fermentable carbon source to a medium containing a fermentable carbon source leads to major changes in the profile of transcription (Thevelein, 1994). Upon a carbon source upshift, among other changes, ribosomal protein gene transcription $-\mathrm{a}$ monitor of the growth potential of yeast cells (Griffioen et al., 1996) - is increased, while transcription of genes under control of the general stress-response pathway is decreased. To study how addition of glucose to yeast cells growing on 
Table 1. Genotypes of S. cerevisiae strains used

\begin{tabular}{|c|c|c|}
\hline Strain & Genotype & Reference \\
\hline W303-1A & $\begin{array}{l}\text { MATa leu2-4,112 ura3-1 trp1-1 his3-11,15 } \\
\text { ade2-1 can1-100 GAL SUC2 }\end{array}$ & $\begin{array}{l}\text { Thomas \& Rothstein } \\
\text { (1989) }\end{array}$ \\
\hline$\Delta m i g 1$ & W303-1A mig1::LEU & J. Winderickx, Leuven \\
\hline MCY 1801 & $\begin{array}{l}\text { MAT } \alpha \text { ade2-101 lys2-801 ura3-52 SUC2 } \\
\text { ssn6 } 69\end{array}$ & Griffioen et al. (1994) \\
\hline SP1 & MATa ade8 his3 leu 2 trp1 ura3 can1 & Cameron et al. (1988) \\
\hline RS13-58A-1 & $\begin{array}{l}\text { SP1 tpk } 1^{\mathrm{w} 1} t p k 2:: \text { HIS3 tpk3::TRP1 } \\
\text { bcy1::LEU2 }\end{array}$ & Toda et al. (1985) \\
\hline$\Delta g p a 2$ & W303-1A gpa2::URA & Colombo et al. (1998) \\
\hline$\Delta g \operatorname{pr} 1$ & W303-1A gpr1::LEU & Kraakman et al. (1999) \\
\hline$\Delta h x k 1 \Delta h k x 2 \Delta g l k 1$ & W303-1A hkx1::HIS hkx2::LEU glk1::LEU & De Winde et al. (1996) \\
\hline$\Delta h x k 1 \Delta h k x 2$ & W303-1A hkx1::HIS hkx2::LEU & De Winde et al. (1996) \\
\hline$\Delta m s n 2 \Delta m s n 4$ & W303-1A msn2::HIS3 msn4::TRP1 & Estruch \& Carlson (1993) \\
\hline ENY.WA-1B & MATa leu 2 trp1 ura3 & Boles et al. (1993) \\
\hline EBY23 & ENY.WA-1B pgi1:: URA3 & Boles et al. (1993) \\
\hline YSH312 & W303-1a tps1A::TRP1 hxk2::LEU2 & Hohmann et al. (1993) \\
\hline Y260 & MATа ura3-52 rpb1-1 & Nonet et al. (1987) \\
\hline
\end{tabular}

(a) $2 \%$ Glucose
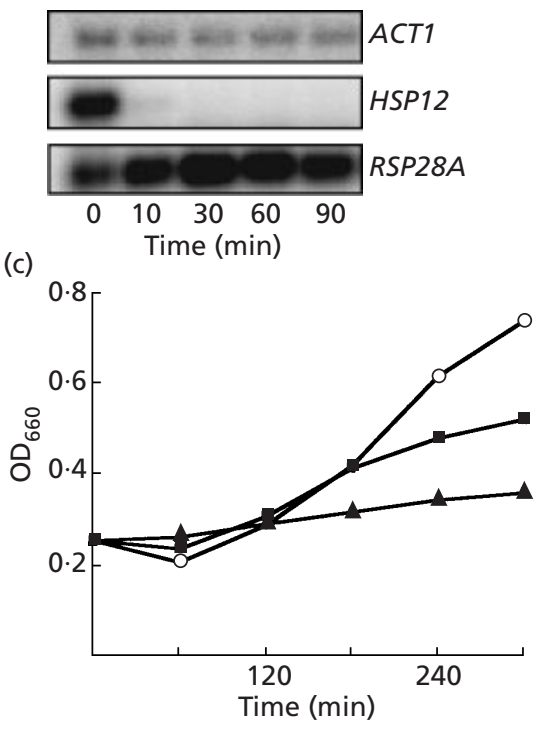

(b) $0.02 \%$ Glucose

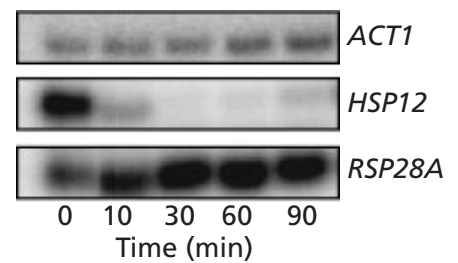

(d) W303-1a

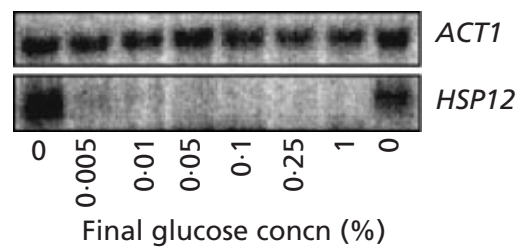

Fig. 1. Glucose-dependent decrease in HSP12 mRNA. (a, b) Northern analysis of mRNA levels for HSP12, RPS28A and ACT1 after addition of glucose to a final concentration of $2 \%$ (a) or $0.02 \%$ (b) to cells pre-grown on YNB/glycerol. (c) Growth curve of wild-type cells pre-grown on YNB/glycerol after addition of glucose to a final concentration of $2 \%(\bigcirc)$ or $0.02 \%$ $(\boldsymbol{\square})$, or water $(\boldsymbol{\Lambda})$ as a control. (d) Northern analysis of mRNA levels for HSP12 and ACT1 30 min after addition of glucose in various concentrations to cells pre-grown on YP/ethanol.

a non-fermentable carbon source causes repression of the general stress-responsive gene HSP12, we performed carbon-source-shift experiments. Cells were pre-grown on a medium containing glycerol as a carbon source, and in early exponential phase glucose was added to a final concentration of $2 \%$. This experimental condition causes a switch from non-fermentative to fermentative growth (Thevelein, 1994). In a wild-type yeast strain (W303-1A) this leads to a loss of approximately $95 \%$ of HSP12 mRNA within $30 \mathrm{~min}$ and a rapid increase in the expression level of the ribosomal protein gene RPS28A (Fig. 1a). Remarkably, however, addition of glucose to a final concentration of only $0.02 \%$ also caused a rapid change in HSP12 and RPS28A mRNA levels (Fig. 1b). This low amount of glucose evokes a growth signal (Fig. 1c), although the yeast cells do not switch their metabolism to fermentation. This switch occurs at glucose concentrations above $0 \cdot 25 \%$ (Meijer et al., 1998), which is much higher than the concentrations used in our experiments. The extreme glucose sensitivity 
(a) $4 \operatorname{mig} 1$

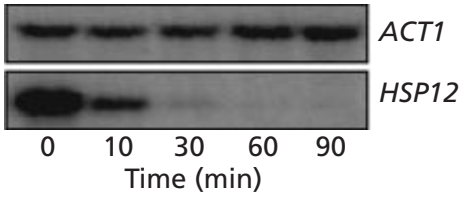

(b) $4 s s n 6$

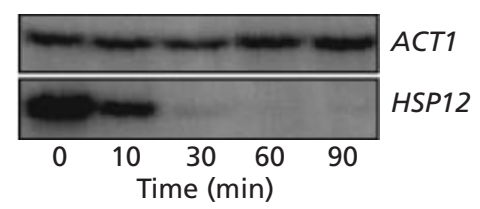

Fig. 2. The main glucose-repression pathway does not mediate glucose-dependent decrease in HSP12 mRNA. Northern blot analysis of mRNA levels for HSP12 and ACT1 after addition of glucose to a final concentration of $2 \%$ to cells pre-grown on YNB/glycerol in a $\Delta m i g 1$ strain (a) and a $\Delta s s n 6$ strain (b). of HSP12 mRNA levels was confirmed in an experiment in which different concentrations of glucose were used. Final glucose concentrations as low as $0.005 \%$ were found to repress HSP12 expression (Fig. 1d). Experiments using another non-fermentable growth medium (YP/ethanol) revealed a similar glucose repression of HSP12 (data not shown).

\section{The main glucose-repression pathway is not involved in glucose repression of $H S P 12$}

Although the low glucose concentration at which HSP12 repression occurs renders it unlikely that the main glucose-repression pathway plays a part, we investigated the effect of MIG1 and SSN6 disruptions on the regulation of HSP12 expression. Addition of $0.02 \%$ glucose to $\Delta m i g 1$ and $\Delta s s n 6$ mutant cells grown on $\mathrm{YNB} /$ glycerol medium caused a repression similar to that seen in wild-type cells (data not shown). This result was anticipated since repression via Mig1p has previously been shown not to occur at glucose concentrations lower than $0 \cdot 2 \%$ (Ozcan et al., 1997; Meijer et al., 1998). To assess the possible involvement of the main glucose-repression pathway in HSP12 gene regulation, the effect of $2 \%$ glucose was also examined. As is clear from the data presented in Fig. 2, HSP12 repression occurred similarly as in wild-type cells, ruling out a role for the main glucose-repression pathway in repression of HSP12.

\section{CAMP-dependent activation of PKA is not required for repression of HSP12}

Addition of glucose to a non-fermenting yeast culture gives rise to a fast increase in the intracellular level of cAMP, which leads to activation of PKA activity (Thevelein, 1994). PKA activity is an important factor in the regulation of HSP12 expression under stress conditions (Varela et al., 1995; Siderius et al., 1997; Gorner et al., 1998). We therefore examined whether activation of PKA via increase in the cAMP level might also be the mechanism underlying repression of HSP12 by glucose. However, in agreement with data previously obtained by Beullens et al. (1988), also under our culture conditions, addition of glucose to a final concentration of $0.02 \%$ did not cause a spike in the cellular cAMP level (data not shown).

To further investigate the putative role of PKA activity in glucose repression of HSP12, we made use of the wimp mutant $\left(t p k 1^{\mathrm{w} 1} \Delta t p k 2 \Delta t p k 3 \Delta b c y 1\right)$, which has a low constitutive level of PKA activity (Cameron et al.,

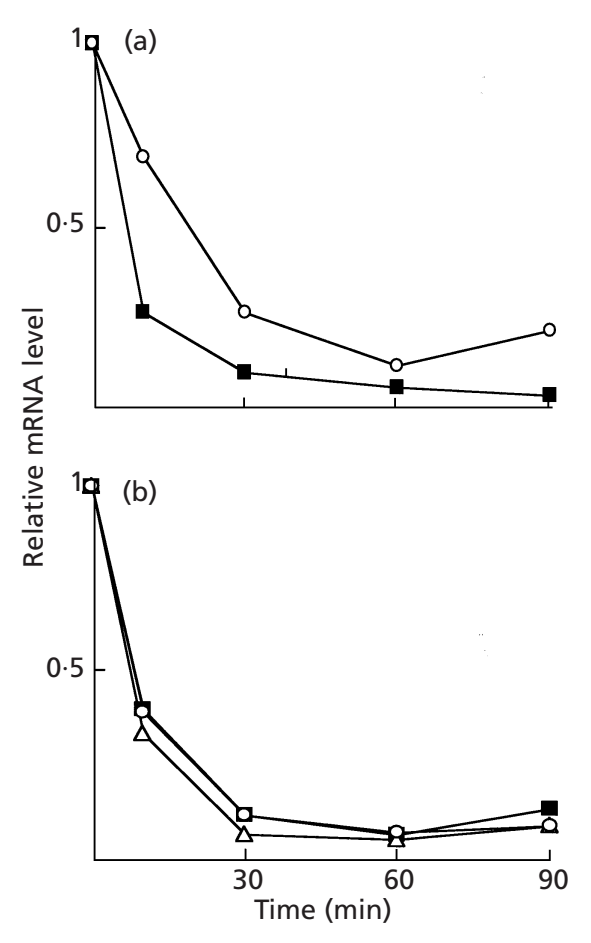

Fig. 3. $C A M P / P K A$ pathway mutants do not affect glucosedependent decrease in HSP12 mRNA. (a) Representation of Northern analysis of HSP12 mRNA levels relative to ACT1 after addition of glucose to a final concentration of $0.02 \%$ to wildtype $(\boldsymbol{\square})$ or tpk $1^{\mathrm{w} 1} \Delta \operatorname{tpk} 2 \Delta t p k 3 \Delta b c y 1(O)$ cells pre-grown on YNB/glycerol. (b) Representation of Northern analysis of HSP12 mRNA levels relative to $A C T 1$ after addition of glucose to a final concentration of $0.02 \%$ to W303-1a ( $\mathbf{\square}), \operatorname{\Delta gpr1}(\triangle)$ or $\Delta g p a 2(\bigcirc)$ cells pre-grown on YNB/glycerol.

1988). In a $0.02 \%$ glucose-shift experiment, HSP12 levels appeared to drop in this strain as in wild-type cells (Fig. 3a), confirming that cAMP-dependent activation of PKA is not involved in glucose repression of this gene. This result also indicates that direct activation of the catalytic subunits Tpk $2 p$ or Tpk3p, which may occur via the FGM pathway (Crauwels et al., 1997), is not essential for glucose repression of HSP12.

Recently, the glucose receptor Gpr1p has been identified (Yun et al., 1998; Kraakman et al., 1999); it is coupled to the heterotrimeric G-protein Gpa2p. It has been postulated that Gpa2p rather than Ras2p (Jiang et al., 1998) is implicated in glucose activation of adenylate cyclase and hence PKA activity (Kubler et al., 1997; Lorenz \& Heitman, 1997; Colombo et al., 1998; Kraakman et al., 1999). We wished to determine whether the Gpr1p- 
(a) $\Delta h x k 1 \Delta h x k 2 \Delta g l k 1$

$0.02 \%$ glucose

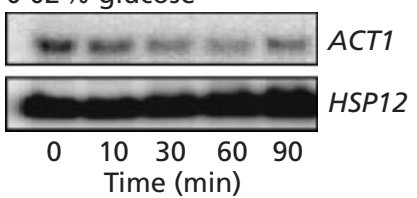

(c) $\Delta h x k 1 \Delta h x k 2$

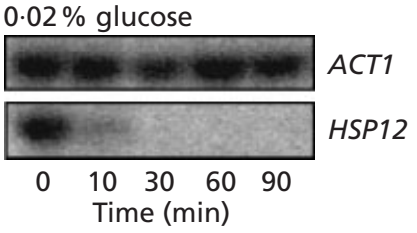

(d) $\Delta h x k 1 \Delta h x k 2$

$0.02 \%$ fructose

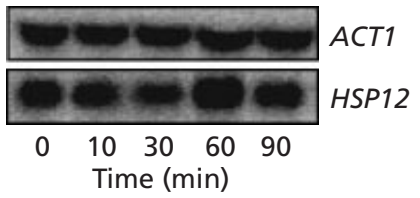

(b) W303-1A

$0.02 \%$ 6-deoxyglucose

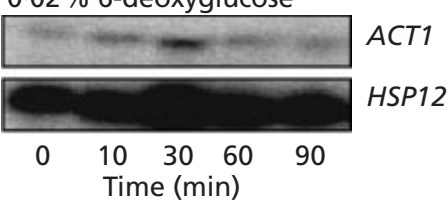

(e) $\Delta$ pgil

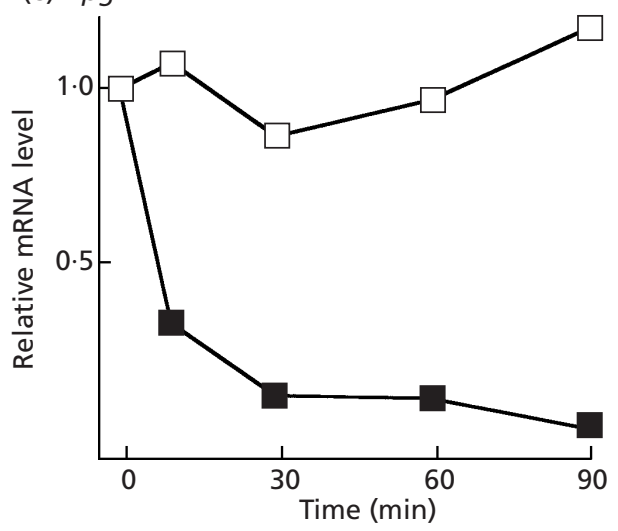

Fig. 4. Glucose phosphorylation is essential for glucose-dependent decrease in HSP12 mRNA. (a) Northern analysis of mRNA levels for HSP12 and ACT1 after addition of glucose to a final concentration of $0.02 \%$ to $\Delta h \times k 1 \Delta h \times k 2 \Delta g / k 1$ cells pre-grown on YNB/glycerol. (b) Northern analysis of mRNA levels for HSP12 and ACT1 after addition of 6-deoxyglucose to a final concentration of $0.02 \%$ to W303-1A cells pre-grown on YNB/glycerol. (c, d) Northern analysis of mRNA levels for HSP12 and ACT1 after addition of glucose (c) or fructose (d) to a final concentration of $0.02 \%$ to $\Delta h x k 1 \Delta h x k 2$ cells pregrown on YNB/glycerol. (e) Representation of Northern analysis of HSP12 mRNA levels relative to ACT1 after addition of glucose $(\boldsymbol{\square})$ or fructose $(\square)$ to a final concentration of $2 \%$ to $\Delta$ pgi1 cells pre-grown on YNB/ethanol.

Gpa2p system might also be involved in glucose repression as observed for the HSP12 gene. The data presented in Fig. 3(b) show that this is not the case; HSP12 repression in both $\Delta g p r 1$ and $\Delta g p a 2$ cells was the same as for the wild-type. Hence we can also exclude the possibility that an unidentified factor downstream of the glucose receptor Gpr1p plays a part in glucose repression of HSP12.

\section{Formation of glucose 6-phosphate is essential for glucose repression of $H S P 12$}

A key question in understanding the molecular mechanism underlying repression of HSP12 by low concentrations of glucose is whether glucose acts as a signalling molecule itself or whether it needs to be metabolized to produce the actual signal. Sugar phosphorylation is the first step in glucose metabolism and we found evidence that this step is essential for repression of HSP12. We used a yeast strain in which the genes for all three sugarphosphorylating enzymes are deleted: $\Delta h x k 1 \Delta h \times k 2-$ $\Delta g l k 1$. Addition of glucose to a final concentration of $0.02 \%$ did not cause a drop in HSP $12 \mathrm{mRNA}$ level in this strain (Fig. 4a). Moreover, addition of the glucose analogue 6-deoxyglucose, which can be taken up by the yeast cell but cannot be phosphorylated, did not cause HSP12 repression in wild-type cells (Fig. 4b). These data strongly suggest that formation of glucose 6-phosphate is required for glucose repression of HSP12. However, an alternative interpretation of our findings could be that binding of the substrate glucose to hexokinase may induce a regulatory function of this protein. Indeed, a regulatory role for $\mathrm{Hxk} 2 \mathrm{p}$ has been postulated previously (De Winde et al., 1996; Herrero et al., 1998; Randezgil et al., 1998). Therefore, we aimed to confirm the importance of sugar phosphorylation for signalling, by using a strain in which both HXK1 and HXK2 are deleted. The kinase still present, Glk1p, can phosphorylate glucose, but not fructose. Addition of a low amount of glucose to a $\Delta h x k 1 \Delta h x k 2$ strain caused repression of HSP12 (Fig. 4c), whereas addition of the same amount of fructose did not (Fig. 4d). These data support our hypothesis that formation of glucose 6phosphate and not the activity of Hxk2p is essential for HSP12 repression by low glucose concentrations.

Another way to analyse the importance of glucose 6phosphate as a potential mediator of regulation is by the use of a $\Delta$ pgi1 strain. In this strain the enzyme catalysing the conversion of glucose 6-phosphate into fructose 6phosphate is absent. Addition of glucose to this strain causes formation of glucose 6-phosphate but no further metabolism can take place (Corominas et al., 1992). Under these conditions HSP12 was found to be repressed (Fig. 4e). In the control experiment fructose was added. This sugar can be further metabolized, but it cannot be converted into glucose 6-phosphate and, consistent with our model, it does not cause repression.

It could be argued that the effect of the $\Delta p g i 1$ mutation on glucose signalling is due to the inability to form trehalose 6-phosphate (from glucose 6-phosphate) rather than the lack of glucose 6-phosphate itself. 
(a) W303-1A

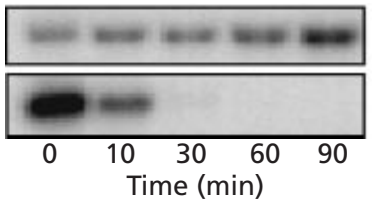

(b) $\Delta m s n 2 \Delta m s n 4$

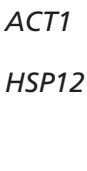

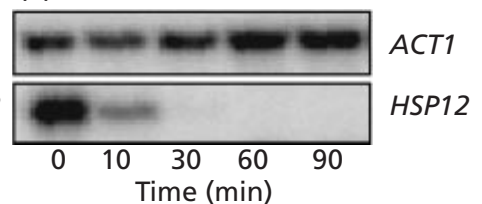

Fig. 5. General stress-responsive transcriptional regulators do not affect the glucose-dependent decrease in HSP12 mRNA. Northern blot analysis of mRNA levels for HSP12 and ACT1 after addition of glucose to a final concentration of $0.02 \%$ to W303-1A (a) or $\Delta m s n 2 \Delta m s n 4$ (b) cells pre-grown on YNB/glycerol.
Therefore we examined a mutant with a TPS1 deletion. In fact, we used a $\Delta t p s 1 \Delta h x k 2$ double mutant, since a $\Delta t p s 1$ mutant rapidly dies on glucose unless hexokinase activity is decreased (Hohmann et al., 1993). Addition of glucose to this $\Delta t p s 1 \Delta h x k 2$ strain caused repression of HSP12 to the same level as in the wild-type (data not shown).

Taken together, the results of these experiments led us to conclude that formation of glucose 6-phosphate, but no further metabolism into glycolysis, is essential for the observed repression of HSP12 by low amounts of glucose. So far it is unknown how this metabolite may mediate signal transmission in yeast.

\section{Msn2p and Msn4p do not play a role in glucose repression of HSP12}

In order to link the role of glucose 6-phosphate as a mediator of repression to a possible control region in the HSP12 promoter, we investigated whether known transcriptional regulators binding to this promoter might be involved in this process. Transcriptional activation of general stress-responsive genes is mediated by the transcriptional regulators $M s n 2 p$ and $M s n 4 p$, which bind to STREs (Martinez-Pastor et al., 1996; Schmitt \& McEntee, 1996). In the HSP12 promoter five such STREs are present (Varela et al., 1995). Under conditions of low PKA activity, Msn2/4p are mainly present in the nucleus, in contrast to conditions where PKA activity is high. We assumed therefore that $M s n 2 / 4 p$ are present in the nucleus during growth on glycerol and translocate to the cytosol when glucose is added, which might cause the drop in HSP12 expression. To test this idea, $0.02 \%$ glucose was added to a glycerol-grown culture of a $\Delta m s n 2 \Delta m s n 4$ strain. Surprisingly, this strain displayed normal expression of HSP12 during growth on glycerol and, moreover, glucose addition caused normal repression of HSP12 (Fig. 5). These data indicate that Msn2/4p are dispensable for the carbon-source-dependent regulation of HSP12 expression.

\section{An STRE in the HSP12 promoter is involved in glucose repression}

Since for several yeast genes glucose-dependent regulation of expression has been shown to be under posttranscriptional control (Lombardo et al., 1992; Cereghino \& Scheffler, 1996; Scheffler et al., 1998), we investigated whether HSP12 mRNA stability might be affected by addition of glucose. Initially we attempted to use a strain which carries a temperature-sensitive
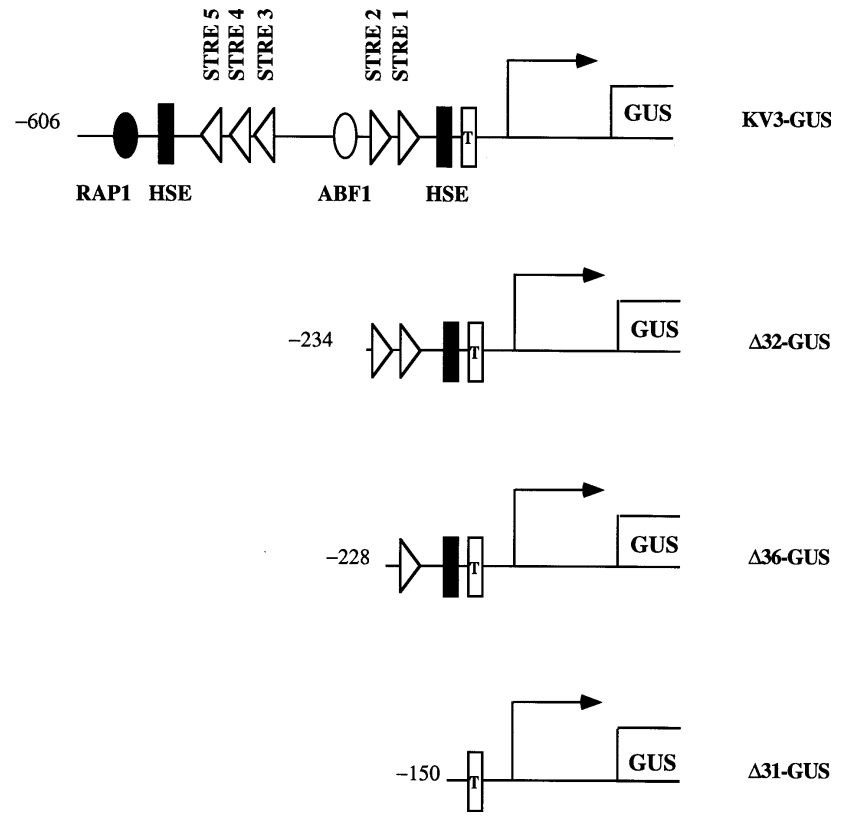

$\triangle 31-G U S$

Fig. 6. Schematic representation of the HSP12-promoterGUS constructs KV3-GUS, $\Delta 32-$ GUS, $\Delta 36-$ GUS and $\Delta 31-G U S$. Putative Rap1p- and Abf1p-binding sites are depicted. Heatshock elements are indicated by HSE, STRE sequences are depicted and a TATA-box is indicated by T.

mutation in the RNA polymerase II subunit Rpb1p (Nonet et al., 1987), which immediately after a shift to the restrictive temperature displays a $95 \%$ transcriptional arrest. We measured the rate of decay of HSP12 mRNA at the restrictive temperature in the presence or absence of glucose, but we could not detect a clear difference. This result may be interpreted as an indication that glucose addition does not cause destabilization of HSP12 mRNA.

In order to discriminate between the possibilities that glucose addition might affect turnover of HSP12 mRNA or transcription of the HSP12 gene, we analysed HSP12promoter-GUS fusion constructs. Fusion of the HSP12 promoter and the GUS reporter has occurred at the ATG so that all fusion genes contain only the leader sequence of the HSP12 gene. Thus, if upon glucose addition post-transcriptional regulation occurs, we would expect the same effect on different fusion-gene mRNAs, irrespective of the promoter region present in the respective constructs. We compared the glucose regulation of the fusion gene containing the full promoter (KV3-GUS, see Fig. 6) with $\Delta 31-$ GUS, in which a 
(a) KV3-GUS

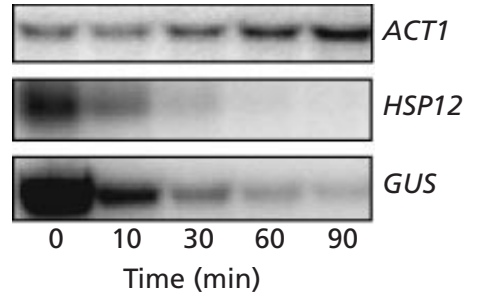

(c) $\Delta 32-$ GUS

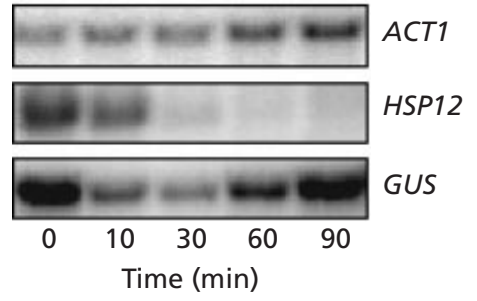

(b) $\Delta 31-G U S$

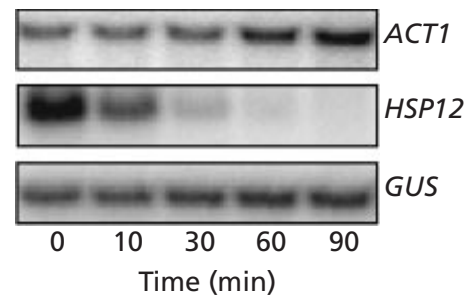

(d) $\Delta 36-$ GUS

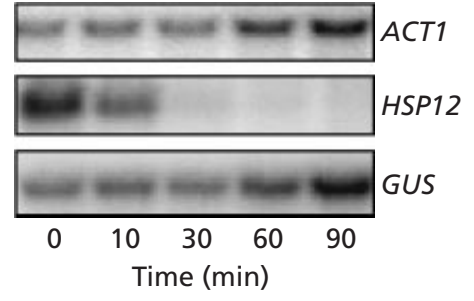

Fig. 7. An STRE is involved in glucose repression of the HSP12 GUS constructs. Northern blot analysis of mRNA levels for GUS, HSP12 and ACT1 after addition of glucose to a final concentration of $0.02 \%$ to W303-1A cells containing the KV3-GUS (a), $\Delta 31-G U S$ (b), $\Delta 32-$ GUS (c) or $\Delta 36-$ GUS (d) construct pre-grown on YNB/glycerol. truncated fragment of the HSP12 promoter, containing no known transcriptional regulatory elements, was fused to a GUS-reporter gene. Expression of the $\Delta 31$ reporter gene appeared not to be subject to glucose repression, whereas the KV3-GUS construct displayed the same regulatory characteristics as the HSP12 gene itself (Fig. 7a, b). Thus, we can conclude that the drop in HSP12 mRNA level after addition of glucose is not caused by post-transcriptional regulation, but is accomplished by a transcriptional repression.

In an attempt to identify the promoter element(s) involved in glucose repression, we made use of other GUS-reporter constructs (see Fig. 6). The construct $\Delta 32-$ GUS lacks several known transcriptional-regulator-binding sites present in the full promoter. Nonetheless, when the transformant strain harbouring this construct was subjected to a carbon-source-shift experiment, it showed a substantial decrease in the level of HSP12 mRNA after $30 \mathrm{~min}$ (Fig. 7c). On the other hand, another construct, $\Delta 36-$ GUS, in which the more upstream of the two proximal STREs present in the $\Delta 32-$ GUS construct is deleted, did not show a decrease in GUS-mRNA level $30 \mathrm{~min}$ after addition of $0.02 \%$ glucose (Fig. 7d). It is clear from Fig. 7 that KV3-GUS expression differs from that of $\Delta 32-$ GUS at later time points (see Discussion). These results led us to conclude that the STRE located between -234 and -228 is essential for the regulation of the transient glucose repression of the HSP12 gene.

\section{DISCUSSION}

The studies presented in this paper demonstrate that expression of HSP12 is not only controlled by stressresponsive signalling, but is also regulated by glucosedependent mechanisms.

Addition of very low amounts of glucose $(0.02 \%)$ to non-fermenting yeast cells appeared to cause rapid repression of HSP12 and induction of RPS28A expression. Repression by low concentrations of glucose has previously also been demonstrated for the gluconeogenic genes FBP1 and PCK1 (Yin et al., 1996). Apparently, even a very low amount of glucose is perceived by yeast cells as a growth-triggering signal. We have shown that phosphorylation of the sugar, but no further metabolism into glycolysis, is required for signalling to HSP12 gene expression. The data supporting this conclusion are that (i) glucose does not cause HSP12 repression in a $\Delta h x k 1 \Delta h x k 2 \Delta g l k 1$ strain, (ii) fructose does not give rise to HSP12 repression in a $\Delta h x k 1 \Delta \mathrm{hxk} 2$ strain, (iii) 6-deoxyglucose has no repressive effect, and (iv) fructose does not lead to repression in a $\Delta$ pgi1 strain. It is likely therefore that glucose 6phosphate serves at the signalling molecule, as was shown previously for the gluconeogenic genes FBP1 and PCK1 (Yin et al., 1996). However, we cannot exclude the possibility that formation of another non-glycolytic metabolite is involved in repression of HSP12 (Gonçalves \& Planta, 1998)

Previously, partial repression of stress-responsive genes such as CTT1 and SSA3 has been shown to occur after addition of $2 \%$ fructose to $\Delta h x k 1 \Delta h x k 2$ cells, suggesting that formation of glucose 6-phosphate is not essential (Pernambuco et al., 1996). We consider it likely that the difference with our data has to do with the final sugar concentration used. Glucose (or fructose) in fermentable amounts may be sensed through the glucose receptor Gpr1p, which activates the adenylate cyclase pathway via Gpa2p (Kraakman et al., 1999). Kraakman et al. (1999) observed repression of HSP12 to be delayed in a $\Delta g p r 1$ or $\Delta g p a 2$ strain. However, we found repression of HSP12 by low amounts of glucose to be unaffected in $\Delta g p r 1$ or $\Delta g p a 2$ strains. We have not further analysed repression by fermentable amounts of sugar and we are not able to discriminate whether signal transduction under these conditions might be composed of both types of glucose signalling.

The low amounts of glucose causing HSP12 repression, as well as the results obtained with the $\Delta m i g 1$ and $\Delta s s n 6$ 
mutants, indicate that the main glucose-repression pathway does not play a role. In addition, evidence was obtained that neither cAMP-dependent activation of PKA, nor cAMP-independent activation of Tpk $2 \mathrm{p}$ or Tpk3p, underlies the signalling by low amounts of glucose. We cannot exclude the possibility that the activity of Tpk1p (wimp) is modulated under these experimental conditions.

Unexpectedly, the transcription factors Msn2p and Msn4p, which play a predominant part in transcriptional activation of HSP12 under stress conditions (Varela et al., 1995), are not implicated in glucose repression of this gene. This finding fits with the negative results concerning the effect of PKA, since activity of Msn $2 / 4 p$ is largely determined by the cellular PKA activity (Gorner et al., 1998). The level of derepression of HSP12 in glycerol-grown cells is similar in the $\Delta m s n 2 \Delta m s n 4$ strain as compared to the wild-type. This raises the question, presently under study, how activation (derepression) of HSP12 in yeast growing on a non-fermentable carbon source occurs.

Given the fact that $M s n 2 / 4 p$ are dispensable for repression of HSP12 by low glucose concentrations, it is even more surprising that an STRE in the promoter of HSP12 may be the target of this regulation. By making use of HSP12-promoter fusion genes the repression phenomenon could be demarcated to a proximal promoter region between -234 and -228 , where an STRE is located. Although the rapid decrease in GUS-mRNA after glucose addition occurred similarly for the KV3GUS and $\Delta 32-$ GUS constructs, a clear difference could be observed at later time points. This phenomenon needs to be investigated further. We consider it likely that this difference reflects the different promoter regions present in the respective constructs, which may be important for sustained repression or resumption of activation. Note that the KV3-GUS construct contains three distal STREs, which may also play a role in glucose repression of HSP12.

Our results suggest that an as yet unidentified factor mediates transcriptional repression of HSP 12 by glucose. We have preliminary evidence, obtained by bandshift analyses, that such an STRE-binding factor, distinct from Msn2/4p, indeed exists. Other workers have reported discrepancies between the effect of STRE deletions and disruption of MSN2/MSN4 (Ni \& LaPorte, 1995; Parrou et al., 1999). It remains to be elucidated if these findings also reflect the action of (a) novel STRE-binding factor(s).

\section{ACKNOWLEDGEMENTS}

The authors wish to thank Dr Paula Gonçalvez, Annelies van der Meulen and Susanna Lima, who also contributed to this work. They thank Dr J. Winderickx (Katholieke Universiteit, Leuven, Belgium) and Dr R. Young (Whitehead Institute, Cambridge, USA) for providing yeast strains.

\section{REFERENCES}

Beullens, M., Mbonyi, K., Geerts, L., Gladines, D., Detremerie, K., Jans, A. W. \& Thevelein, J. M. (1988). Studies on the mechanism of the glucose-induced cAMP signal in glycolysis and glucose repression mutants of the yeast Saccharomyces cerevisiae. Eur J Biochem 172, 227-231.

Boles, E., Heinisch, J. \& Zimmermann, F. K. (1993). Different signals control the activation of glycolysis in the yeast Saccharomyces cerevisiae. Yeast 9, 761-770.

Cameron, S., Levin, L., Zoller, M. \& Wigler, M. (1988). cAMPindependent control of sporulation, glycogen metabolism, and heat shock resistance in S. cerevisiae. Cell 53, 555-566.

Cereghino, G. P. \& Scheffler, I. E. (1996). Genetic analysis of glucose regulation in Saccharomyces cerevisiae: control of transcription versus mRNA turnover. EMBO J 15, 363-374.

Colombo, S., Ma, P. S., Cauwenberg, L. \& 8 other authors (1998). Involvement of distinct G-proteins, Gpa2 and Ras, in glucoseand intracellular acidification-induced cAmp signalling in the yeast Saccharomyces cerevisiae. EMBO J 17, 3326-3341.

Corominas, J., Clotet, J., Fernandez-Banares, I., Boles, E., Zimmermann, F. K., Guinovart, J. J. \& Arino, J. (1992). Glycogen metabolism in a Saccharomyces cerevisiae phosphoglucose isomerase (pgil) disruption mutant. FEBS Lett 310, 182-186.

Crauwels, M., Donaton, M. C. V., Pernambuco, M. B., Winderickx, J., De Winde, J. H. \& Thevelein, J. M. (1997). The Sch9 protein kinase in the yeast Saccharomyces cerevisiae controls cApk activity and is required for nitrogen activation of the fermentablegrowth-medium-induced (FGM) pathway. Microbiology 143, 2627-2637.

De Winde, J. H., Crauwels, M., Hohmann, S., Thevelein, J. M. \& Winderickx, J. (1996). Differential requirement of the yeast sugar kinases for sugar sensing in establishing the catabolite-repressed state. Eur J Biochem 241, 633-643.

Estruch, F. \& Carlson, M. (1993). Two homologous zinc finger genes identified by multicopy suppression in a SNF1 protein kinase mutant of Saccharomyces cerevisiae. Mol Cell Biol 13, 3872-3881.

Gancedo, J. M. (1998). Yeast carbon catabolite repression. Microbiol Mol Biol Rev 62, 334-361.

Gonçalves, P. \& Planta, R. J. (1998). Starting up yeast glycolysis. Trends Microbiol 6, 314-319.

Gorner, W., Durchschlag, E., Martinez-Pastor, M. T., Estruch, F., Ammerer, G., Hamilton, B., Ruis, H. \& Schuller, C. (1998). Nuclear localization of the $\mathrm{C} 2 \mathrm{H} 2$ zinc finger protein $\mathrm{Msn} 2 \mathrm{p}$ is regulated by stress and protein kinase A activity. Genes Dev 12, 586-597.

Griffioen, G., Mager, W. H. \& Planta, R. J. (1994). Nutritional upshift response of ribosomal protein gene transcription in Saccharomyces cerevisiae. FEMS Microbiol Lett 123, 137-144.

Griffioen, G., Laan, R. J., Mager, W. H. \& Planta, R. J. (1996). Ribosomal protein gene transcription in Saccharomyces cerevisiae shows a biphasic response to nutritional changes. Microbiology 142, 2279-2287.

Herrero, P., Martinezcampa, C. \& Moreno, F. (1998). The hexokinase 2 protein participates in regulatory DNA-protein complexes necessary for glucose repression of the SUC2 gene in Saccharomyces cerevisiae. FEBS Lett 434, 71-76.

Hohmann, S. (1997). Shaping up: the responses of yeast to osmotic stress. In Yeast Stress Responses, pp. 101-146. Edited by S. Hohmann \& W. H. Mager. Georgetown, TX: R. G. Landes.

Hohmann, S., Neves, M. J., de Koning, W., Alijo, R., Ramos, J. \& Thevelein, J. M. (1993). The growth and signalling defects of the 
ggs1 (fdp1/byp1) deletion mutant on glucose are suppressed by a deletion of the gene encoding hexokinase PII. Curr Genet 23, 281-289.

Jiang, Y., Davis, C. \& Broach, J. R. (1998). Efficient transition to growth on fermentable carbon sources in Saccharomyces cerevisiae requires signaling through the Ras pathway. EMBO J 17, 6942-6951.

Kraakman, L., Lemaire, K., Ma, P. S., Teunissen, A., Donaton, M. C. V., Van Dijck, P., Winderickx, J., de Winde, J. H. \& Thevelein, J. M. (1999). A Saccharomyces cerevisiae G-protein coupled receptor, Gpr1, is specifically required for glucose activation of the cAMP pathway during the transition to growth on glucose. Mol Microbiol 32, 1002-1012.

Kubler, E., Mosch, H. U., Rupp, S. \& Lisanti, M. P. (1997). Gpa2p, a G-protein alpha-subunit, regulates growth and pseudohyphal development in Saccharomyces cerevisiae via a cAMP-dependent mechanism. J Biol Chem 272, 20321-20323.

Lombardo, A., Cereghino, G. P. \& Scheffler, I. E. (1992). Control of mRNA turnover as a mechanism of glucose repression in Saccharomyces cerevisiae. Mol Cell Biol 12, 2941-2948.

Lorenz, M. C. \& Heitman, J. (1997). Yeast pseudohyphal growth is regulated by Gpa2, a $\mathrm{G}$ protein alpha homolog. EMBO J 16, 7008-7018.

Lundin, M., Nehlin, J. O. \& Ronne, H. (1994). Importance of a flanking AT-rich region in target site recognition by the GC boxbinding zinc finger protein MIG1. Mol Cell Biol 14, 1979-1985.

Mager, W. H. \& Planta, R. J. (1991). Coordinate expression of ribosomal protein genes in yeast as a function of cellular growth rate. Mol Cell Biochem 104, 181-187.

Martinez-Pastor, M. T., Marchler, G., Schuller, C., Marchler-Bauer, A., Ruis, H. \& Estruch, F. (1996). The Saccharomyces cerevisiae zinc finger proteins $M s n 2 p$ and $M s n 4 p$ are required for transcriptional induction through the stress response element (STRE). EMBO J 15, 2227-35.

Meijer, M. M. C., Boonstra, J., Verkleij, A. J. \& Verrips, C. T. (1998). Glucose repression in Saccharomyces cerevisiae is related to the glucose concentration rather than the glucose flux. $J$ Biol Chem 273, 24102-24107.

Moskvina, E., Schuller, C., Maurer, C. T., Mager, W. H. \& Ruis, H. (1998). A search in the genome of Saccharomyces cerevisiae for genes regulated via stress response elements. Yeast 14, 1041-1050.

Nehlin, J. O., Carlberg, M. \& Ronne, H. (1991). Control of yeast GAL genes by MIG1 repressor: a transcriptional cascade in the glucose response. EMBO J 10, 3373-3377.

Ni, H. T. \& LaPorte, D. C. (1995). Response of a yeast glycogen synthase gene to stress. Mol Microbiol 16, 1197-1205.

Nonet, M., Scafe, C., Sexton, J. \& Young, R. (1987). Eucaryotic RNA polymerase conditional mutant that rapidly ceases mRNA synthesis. Mol Cell Biol 7, 1602-1611.

Ozcan, S., Vallier, L. G., Flick, J. S., Carlson, M. \& Johnston, M. (1997). Expression of the SUC2 gene of Saccharomyces cerevisiae is induced by low levels of glucose. Yeast 13, 127-137.

Parrou, J. L., Enjalbert, B., Plourde, L., Bauche, A., Gonzalez, B. \& Francois, J. (1999). Dynamic responses of reserve carbohydrate metabolism under carbon and nitrogen limitations in Saccharomyces cerevisiae. Yeast 15, 191-203.

Pernambuco, M. B., Winderickx, J., Crauwels, M., Griffioen, G., Mager, W. H. \& Thevelein, J. M. (1996). Glucose-triggered signalling in Saccharomyces cerevisiae: different requirements for sugar phosphorylation between cells grown on glucose and those grown on non-fermentable carbon sources Microbiology 142, 1775-1782.

Praekelt, U. M. \& Meacock, P. A. (1990). HSP12, a new small heat shock gene of Saccharomyces cerevisiae: analysis of structure, regulation and function. Mol Gen Genet 223, 97-106.

Randezgil, F., Sanz, P., Entian, K. D. \& Prieto, J. A. (1998). Carbon source-dependent phosphorylation of hexokinase PII and its role in the glucose-signaling response in yeast. Mol Cell Biol 18, 2940-2948.

Ronne, H. (1995). Glucose repression in fungi. Trends Genet 11, $12-17$.

Scheffler, I. E., Delacruz, B. J. \& Prieto, S. (1998). Control of mRNA turnover as a mechanism of glucose repression in Saccharomyces cerevisiae. Int J Biochem Cell Biol 30, 1175-1193.

Schmitt, A. P. \& McEntee, K. (1996). Msn2p, a zinc finger DNAbinding protein, is the transcriptional activator of the multistress response in Saccharomyces cerevisiae. Proc Natl Acad Sci USA 93, 5777-5782.

Siderius, M., Rots, E. \& Mager, W. H. (1997). High-osmolarity signalling in Saccharomyces cerevisiae is modulated in a carbonsource-dependent fashion. Microbiology 143, 3241-3250.

Thevelein, J. M. (1991). Fermentable sugars and intracellular acidification as specific activators of the RAS-adenylate cyclase signalling pathway in yeast: the relationship to nutrient-induced cell cycle control. Mol Microbiol 5, 1301-1307.

Thevelein, J. M. (1994). Signal transduction in yeast. Yeast 10, 1753-1790.

Thomas, B. J. \& Rothstein, R. (1989). Elevated recombination rates in transcriptionally active DNA. Cell 56, 619-630.

Toda, T., Uno, I., Ishikawa, T. \& 7 other authors (1985). In yeast, RAS proteins are controlling elements of adenylate cyclase. Cell 40, 27-36.

Treitel, M. A. \& Carlson, M. (1995). Repression by SSN6-TUP1 is directed by MIG1, a repressor/activator protein. Proc Natl Acad Sci USA 92, 3132-3136.

Varela, J. C., Praekelt, U. M., Meacock, P. A., Planta, R. J. \& Mager, W. H. (1995). The Saccharomyces cerevisiae HSP12 gene is activated by the high-osmolarity glycerol pathway and negatively regulated by protein kinase A. Mol Cell Biol 15, 6232-6245.

Yin, Z. K., Smith, R. J. \& Brown, A. J. P. (1996). Multiple signalling pathways trigger the exquisite sensitivity of yeast gluconeogenic mRNAs to glucose. Mol Microbiol 20, 751-764.

Yun, C. W., Tamaki, H., Nakayama, R., Yamamoto, K. \& Kumagai, H. (1998). Gpr1p, a putative G-protein coupled receptor, regulates glucose-dependent cellular cAMP level in yeast Saccharomyces cerevisiae. Biochem Biophys Res Commun 252, 29-33.

Received 2 September 1999; revised 18 October 1999; accepted 17 November 1999. 